\title{
VERDE QUE TE QUIERO VERDE. INCOMPATIBILIDADES ENTRE NUESTRA REALIDAD Y NUESTRA RACIONALIDAD
}

\section{GREEN, HOW I WANT YOU GREEN: INCOMPATIBILITIES BETWEEN OUR REALITY AND RATIONALITY}

\author{
M.EL. Ernesto Herra Castro \\ Universidad Nacional de Costa Rica \\ Luis Navarro Gómez \\ Universidad Nacional de Costa Rica, estudiante Arte y comunicación visual
}

Recibido: 24 de junio, $2015 \bullet$ Aceptado: 14 de agosto, 2015

Resumen: El artículo muestra cómo el tipo de racionalidad de dominio contenida en la modernidad ha impuesto una única forma de nombrar y relacionarnos en nuestro trópico mesoamericano, expreso en una gama exuberantemente de verdes en escala policromática. Lo anterior es posible a partir de una problematización de este tipo de racionalidad para pensar, de manera modesta, en los retos prospectivos que enfrenta la región mesoaméricana para garantizar la producción y reproducción de la vida a futuro.

Palabras clave: Mesoamérica, trópico húmedo, modernidad, colonialidad, color

Abstract: The paper shows how the type of domain contained in modern rationality has imposed a unique way of naming and relates to Mesoamerican humid tropic expressed in a polychromatic range in lushly green. This is possible from a problematization of this type of rationality to think, modestly, in prospective challenges facing the Mesoamerican region to guarantee the production and reproduction of life in the future.

Keywords: Mesoamerica, Humid Tropics, Modernity, Coloniality, Color 


\section{A modo de introducción}

A lo largo del siglo XVI se desarrolló en Francia una álgida discusión respecto a la lengua y el lenguaje, que tuvo como propósito, la mayoría de las veces, buscar el origen de las lenguas. Este interés particular, que tenía por objetivo demostrar la cercanía o la distancia que un pueblo mantenía con la «lengua sagrada inspirada por Dios» (Conrad, 1970, citado por Calvet, 2005, p. 30), impulsó la división de la lengua en dos grandes grupos. Por un lado se ubicaron aquellas que mantenían un vínculo directo con la lengua originaria, denominadas sagradas. En ellas se encuentran el latín, el griego y el hebreo, esta última se consideró la lengua más cercana a la lengua originaria; por otro lado se ubicaron todas las otras lenguas (Calvet, 2005).

La estructuración de este orden étnico tuvo como principio la cercanía o la distancia que una lengua mantuviese con la lengua originaria, modelando un sistema racial que peyorizó e inferiorizó a las otras lenguas, calificadas ahora como «bárbaras», y a los pueblos cuya comprensión del cosmos y relación con él se desarrollaba en ellas. De esta forma cobra sentido el señalamiento realizado por Calvet (2005) en cuanto a que

Las relaciones entre lenguas se conciben como relaciones sociales: hay una jerarquía, con una cima (para unos pocos elegidos) y una base en que se encuentra la masa. Esa organización piramidal de las lenguas, es decir, de los pueblos que las hablan, hace recordar un poco la arquitectura de la torre de Babel, pero sobre todo la organización social de la época (p. 31).

Lo anterior permite identificar cómo el impulso originario de la lingüística por comprender el origen y funcionamiento de la lengua estuvo conducido por el germen ideológico del racismo (Calvet, 2005), el cual organizó, reprodujo y legitimó las relaciones vasallo-feudales ${ }^{1}$ propias del orden romano, y las ha fundamentado filosóficamente a partir de los presupuestos

1 Las relaciones vasallo-feudales fueron un conjunto de prácticas fundamentadas en la sumisión, la lealtad y el respeto que le debía el vasallo a su amo, denominadas Fidélitas, y este último le debía protección y cuidado a quien era de su propiedad: Fides. Este tipo de relaciones de inferioridad/superioridad sobre la línea de lo humano (Grosfoguel, 2011) datan de la constitución de la República de Roma en el 509 a.C., las cuales atraviesan su época de Imperio (27 a.C.-476 d.C.) y se extienden hasta su caída bajo el mando del Imperio otomano en el 711 de nuestra era. 


\section{de la racionalidad ${ }^{2}$ moderna, ${ }^{3}$ lo que le ha permitido a Occidente imponerse $^{4}$} colonialmente sobre las prácticas comunitarias de los pueblos no modernos. ${ }^{5}$ Partiendo del principio señalado en Calvet (2005) en cuanto a que «la lengua evoluciona a la par que el pensamiento se hace más agudo» (p. 39), a la vez que «lengua y pensamiento son estrictamente paralelos [lo que demuestra $\left.q \mathrm{e}^{6}\right]$ los avances de una manifiestan avances del otro, y el carácter inacabado de uno torna imposible el acabamiento de la otra» (p.39), es que queremos pensar en los límites existentes para nombrar, de manera apropiada, la gama diversa e infinita de verdes que nutren y reflejan la vida exuberante de los pueblos que damos forma a nuestras realidades amerindias, indoamericanas, caribeñas y latinas que se expresan en el trópico húmedo ${ }^{7}$ de la región mesoamericana. Lo

2 El tipo de racionalidad contenida en el proyecto de la modernidad está fundamentada en la imposición de un tipo de explicación que determina al objeto sin modificar al sujeto. Este tipo de relación en el proceso de construcción de conocimiento tiene que ver con el tipo de pregunta planteada en la Grecia clásica, la cual es retomada por Occidente durante los siglos XVI y XVII gracias a que los teólogos y filósofos árabes se habían interesado por la noción teológica y filosófica existente en Grecia. Este particular se conoce en la periodización del tiempo histórico occidental como Renacimiento, precisamente porque le permite a Occidente desarrollar un tipo de racionalidad en la que sus presupuestos justifican su superioridad y por ende su imposición colonial sobre los otros, que se ubican como los «barbaros» modernos.

3 La modernidad, que no es otra cosa que el surgimiento de la Europa latino-germánica como centro del sistema-mundo, es el resultado de un largo proceso de imposición colonial que inició a partir de la ofensiva militar de Hispania sobre el mundo árabe situado en el al-Ándalus el 6 de enero de 1492 y continuó con la imposición colonial sobre los pueblos autóctonos de lo que hoy es conocido como América, África, Asia y Oceanía. Es importante señalar aquí que nos distanciamos de la noción pretendida en la teoría occidental de marcar el surgimiento de la modernidad como el momento de surgimiento de un método que hiciese posible materializar las relaciones de dominio contenidas en los presupuestos de la filosofía griega a partir del Discurso del método de Descartes en 1637. Si bien filósofos como Kant o Hegel situaron a la modernidad como el «modo de relación de la razón consigo misma» (Rendón, 2012, p. 47), nos interesa resaltar que esto fue solo posible en Europa gracias al saqueo, la explotación y la miseria de los pueblos «bárbaros», lo que les ha permitido, por primera vez en la historia de la humanidad, situarse como centro del sistema-mundo.

4 Comprendemos por Occidente el conjunto de países de la Europa latino-germánica que se imponen colonialmente en el Anáhuac, el Abya-Yala y el Tawantinsuyu, conocidos desde entonces como Nuevo Mundo, Nueva España o lo que en la actualidad es «la invención de América Latina», como denominó con acierto Mignolo (2007). Lo anterior se ubica entre los años 1492-1870, ya que posterior a esta última fecha se incorporó, en un nuevo rol de opresor, Estados Unidos a la lucha por administrar los territorios coloniales en América, vía exportación de capitales (Menjívar, 1980) en la región centroamericana. A partir de ese momento los linderos geográficos de Europa se ensancharon hasta el otro lado del Atlántico.

5 La distinción establecida por Marx, y retomada por el sociólogo boliviano Juan José Bautista, entre gesellschaft (sociedad) y gemeinschaft (comunidad) caracteriza a lo social como aquello conformado por individuos aislados, ego-ístas, relacionados a través de contratos formales; mientras que lo comunitario tiene, como horizonte de sentido, la producción y reproducción de la vida, razón por la cual las relaciones sociales han intentado constantemente desplazar las relaciones comunitarias desarrolladas por los pueblos no modernos (Bautista, 2010).

$6 \quad$ La negrita es de los autores del presente artículo.

7 Nuestras realidades caribeñas, mesoamericanas, centroamericanas, indoamericanas y amerindias urgen formas propias de nombrar y relacionarse con la inmensa policromía de verdes que se manifiestan en ella, no solo como una urgencia política tras 522 años de sufrir la imposición colonial de Occidente, sino porque de ello depende la posibilidad de agudizar el mismo pensamiento que una vez fuera capaz de imaginar la noción del 
anterior sugiere confrontar los mecanismos de imposición de un tipo específico de conciencia que, como señalara Hegel, ha sido constituida intersubjetivamente y que esta constitución es de carácter autorreflexiva (Rendón, 2012).

Partiendo del hecho histórico de que la imposición colonial de la Europa latino-germánica modificó, aplastó, mas no colapsó las relaciones comunitarias de los pueblos autóctonos del Anáhuac, el Abya-Yala y el Tawantinsuyu, mas configuró la subjetividad-objetiva, es decir, el conjunto de relaciones que se expresan en el ámbito de la vida en común (Rendón, 2012) y que, por lo tanto, afectó las formas intersubjetivas de constitución de la conciencia, es que se piensa en la generación de sospechas más que de respuestas que tiendan a problematizar el tipo de racionalidad que ha naturalizado, por la vía de la objetivación, las relaciones de inferioridad/superioridad con que se expresa, de forma concreta, el racismo ${ }^{8}$ en la vida y en la historia de los pueblos no modernos. ${ }^{9}$ Se trata de avanzar hacia la agudeza y el acabamiento de nuestro propio pensamiento.

\section{Una problematización de partida más allá del color}

El tipo de racionalidad contenida en la modernidad solo ha sido capaz de aplicarse hegemónicamente a escala planetaria, producto de la imposición colonial de Occidente tanto sobre los pueblos del subcontinente latinoamericano, como sobre África, Asia y Oceanía.

Dussel, Galeano, Quijano, Bautista y un grupo importante de autoras y autores del Tercer Mundo ${ }^{10}$ comparten la tesis de que no existiría la

0 (cero), en el que está estructurado el código binario que hace posible la producción y reproducción de las tasas de ganancia y utilidad capitalista, horizonte de sentido contenido en la modernidad.

8 Parafraseamos al sociólogo boliviano Juan José Bautista (2010) cuando señaló que el orden jerárquico racial que conocemos en la actualidad es producto de las relaciones de dominio con que se ha impuesto la modernidad. Sin embargo, es importante recalcar aquí que el orden racial estructurado en la modernidad no tiene una manifestación práctica o real previo a 1492.

9 Si bien es cierto que la modernidad ha atropellado a todos los pueblos que han surgido posterior a 1492, esto no quiere decir que el proyecto de brutalidad colonial con que se ha impuesto Occidente como centro de dominio, control e imposición colonial en el sistema-mundo moderno le es propio a la especie humana.

10 La noción de Tercer Mundo ha sido utilizada por sectores liberales y conservadores de las ciencias sociales occidentales u occidentalizadas para designar una amplia zona geográfica carente de condiciones materiales para alcanzar el «desarrollo»; otra denotación tiene que ver con el interés ideológico de señalar a aquellos países que en el contexto de la guerra Fría no asumieron ninguna posición política durante esta coyuntura global; otro, que es desde donde se parte en la presente reflexión, tiene que ver con aquellos pueblos, comunidades, naciones y nacionalidades que han sido incorporados al sistema-mundo moderno a través de dinámicas de imposición colonial. Esta última acepción tuvo auge durante las décadas del cincuenta y del sesenta del siglo XX, impulsando diversos procesos de liberación nacional en África y América Latina. Lo anterior ha socavado las condiciones materiales con que se ha reproducido e impuesto la modernidad desde 1492. 
posibilidad de haberse dotado de las bases materiales sobre las que está estructurado el sistema-mundo moderno si no fuese por la imposición colonial de la Europa latino-germánica en este. Un señalamiento crítico se encuentra en la carta escrita por Carlos Marx a Pavel Vasilievich Annekov, fechada el 28 de diciembre de 1846, en la que Marx pensó la esclavitud como una de las principales categorías sobre la que está estructurada la modernidad. Según Marx (1976), y nos permitimos citar en extenso,

La esclavitud directa es un pivote de nuestro industrialismo actual, lo mismo que las máquinas, el crédito, etc. Sin la esclavitud, no habría algodón, y sin algodón, no habría industria moderna. Es la esclavitud lo que ha dado valor a las colonias, son las colonias lo que ha creado el comercio mundial, y el comercio mundial es la condición necesaria de la gran industria mecanizada. Así, antes que la trata de negros, las colonias no daban al mundo viejo más que unos pocos productos y no cambiaron visiblemente la faz de la tierra. La esclavitud, es, por tanto, una categoría económica de la más alta importancia. Sin la esclavitud, Norteamérica, el país más desarrollado, se transformaría en país patriarcal. Si se borra a Norteamérica del mapa del mundo, tendremos la anarquía, la decadencia absoluta del comercio y de la civilización moderna. Pero hacer desaparecer la esclavitud equivaldría a borrar a Norteamérica del mapa del mundo. La esclavitud es una categoría económica y por eso se observa en cada nación desde que el mundo es mundo. Los pueblos modernos sólo han sabido disfrazar la esclavitud en sus propios países e importarla al nuevo mundo (p. 536).

El secuestro de la pregunta de la Grecia clásica (siglos IV y III a.C.) por parte de Occidente ha permitido la imposición de un sistema planetario estructurado de manera vertical a partir de un orden étnico complejo, cuya línea divisoria de lo humano es la raza (Grosfoguel, 2011). Esta línea divisoria, justificada por Aristóteles como la ley natural, ha garantizado, hasta la actualidad, la reproducción de las relaciones de dominio contenidas en el tipo de pregunta planteada por Grecia en el tiempo en que esta era una colonia egipcia, el sistema-mundo giraba alrededor de China y Grecia tenía la necesidad de resistir los constantes embates militares de sus enemigos de ultramar.

Este tipo de racionalidad, que le permitió a la Europa latino-germánica renacer de entre sus cenizas, fue posible de desarrollar e imponer 
hegemónicamente en términos planetarios gracias a que filósofos y teólogos del Imperio Otomano como Averroes y Avicena se interesaron por la filosofía griega y la traducción de textos clásicos. Lo anterior les permitió plantearse nuevas formas de proceder, ya que la traducción griega de los textos fundantes de la tradición cristiana le asignaba un papel central al ser humano como hacedor de conocimiento, como sujeto, y no solo como un objeto para ser llenado de contenido, tal como había sido desarrollado por el orden teológico medieval a partir de relaciones sociales organizadas en torno a la verticalidad vasallo-feudal.

El viraje establecido por la Europa latino-germánica a partir de las primeras décadas del siglo XIV le permitió a esta dotarse de los recursos intelectuales y la justificación cultural para imponerse sobre otros pueblos. Sin embargo, la posibilidad de hacerlo solo era avanzando hacia el este a través del Principado de Moscú o del territorio controlado por el Imperio otomano. Una y otra no eran posibles desde el punto de vista bélico, primero porque el Imperio otomano controlaba su territorio desde el 711 de nuestra era; segundo porque Europa no era más que una cultura periférica del sistema-mundo que giraba en torno a China sin nada que vender y, por ende, sin recursos materiales que le permitieran jugar un papel de mayor protagonismo que el que hasta entonces había ocupado (Dussel, 2011). La posibilidad de jugar ese papel fue solo posible a partir de la adquisición por parte del Vaticano de varios mapas que los navegantes chinos habían trazado durante sus años de navegación alrededor del globo. ${ }^{11}$

La imposición colonial que la Europa latino-germánica hizo sobre el Anáhuac, el Abya-Yala y el Tawantinsuyu, posteriormente el Nuevo Mundo, la Nueva España o lo que hoy es conocido como América latina, fue producto de la fuerza militar con que el caballo, los perros de caza, el arcabuz y los gérmenes se abrieron paso en medio de una cultura milenaria que tenía

11 Durante la Edad Media, el predominio que tuvo la Iglesia cristiana en el centro de las relaciones sociales, principalmente en el campo del conocimiento, definió, desde sus presupuestos, el hecho de que los navegantes cristianos debían contar con un límite marítimo para no llegar a caer en una especie de abismo que estas gentes se imaginaban, dado que al ser la Tierra plana y solo una parte del universo, entre una cosa y otra debía existir algo así como un despeñadero. Por el contrario, los chinos ya tenían mapeado y cartografiado el planeta desde 1418 y fue la dotación de estos mapas por parte del Vaticano lo que les permitió a los reyes católicos de España apostar por el proyecto de dominio colonial sobre aquellas tierras hasta donde el mundo latino no había llegado. Sobre lo anterior, el sociólogo puertorriqueño Ramón Grosfoguel tiene señalamientos muy interesantes y pertinentes que valdría la pena revisar. 
otras formas intersubjetivas de comprender el mundo y relacionarse con él. Lo anterior deja en evidencia que no fue producto de una razón superior, sino de la brutalidad con la que la Europa latino-germánica se garantizó la centralidad del sistema-interregional ${ }^{12}$ que comenzó a ser sistema-mundo por primera vez ante la incorporación, vía imposición militar, de los pueblos, las comunidades y las civilizaciones preexistentes en «América».

Queda así en evidencia que más allá del criterio tautológico utilizado por Occidente para calificar de superior el tipo de racionalidad que le ha permitido imponerse a escala global, ha sido la imposición colonial que Occidente ${ }^{13}$ ha mantenido en el Tercer Mundo lo que le ha permitido garantizar para sí la base material con la que ha garantizado ubicarse en el centro del sistema-mundo.

Lo anterior es un señalamiento problemático que contiene un proyecto político en tanto horizonte de sentido para los países del Tercer Mundo, quienes no hemos tenido la libertad y/o posibilidad de comprender y relacionarnos con el cosmos a partir de nuestras propias realidades ubicadas en nuestro tiempo-espacio. ${ }^{14} \mathrm{Al}$ contrario, hemos sido modeladas y modelados en una lógica con un tiempo histórico ajeno a nuestro entorno, a nuestra realidad inferiorizada y coloniazada que no logra dar cuenta ni teórica ni

12 Sobre este elemento el sociólogo boliviano Juan José Bautista hizo un llamado de atención en tanto que no se puede hablar de sistema-mundo antes de 1492, sino de un sistema-interregional, ya que el sistema-mundo anterior a la modernidad/colonialidad «abarcaba desde Marruecos, el mundo berebere del norte africano, hacia el oriente, atravesando la Mesopotamia, Kabul, la India y hasta la China» (Bautista, 2010, p. 100).

13 El surgimiento de los Estados Unidos como nación independiente el 4 de julio de 1776, a partir de la confrontación de las trece colonias con el Imperio británico, fue un momento de quiebre determinante en la expansión de los linderos que Occidente había definido. Otro momento de quiebre fue el año de 1823 con la aparición de la Doctrina Monroe, donde los Estados Unidos definieron una política colonial para el sub continente latinoamericano ante la confrontación entre las potencias coloniales (Inglaterra y Francia) de la época. Asimismo, otro momento de quiebre, y quizá el definitivo en el afianzamiento de las condiciones estructurales para garantizar la imposición colonial de los Estados Unidos sobre la región latinoamericana, ocurrió en el año de 1870, año en el que se inició la dinámica de exportación de capitales de los Estados Unidos hacia El Salvador. Sobre este particular, Ernesto Herra se encuentra preparando un pequeño libro reflexivo a partir de los aportes de Wallerstein para comprender el sistema-mundo situado desde nuestras posicionalidades latinoamericanas, amerindias, indoamericanas, negras, mesoamericanas, caribeñas y centroamericanas, que piensa las líneas epistémicas del sociólogo estadounidense Immanuel Wallerstein en clave anticolonial.

14 Sobre la base de confrontar el esquema metódico con que la racionalidad moderna limita, explica y le da sentido no solo al objeto, sino a la delimitación temporal impuesta por esta, el libro Pensar y poder de Hugo Zemelman problematiza y propone algunas líneas para construir preguntas orientadas a «concebir la construcción del conocimiento desde un espacio más amplio que el de la premisa teórica como lo propio de la colocación del sujeto» (Zemelman, 2012, p. 106). De esta forma, los presupuestos, los cánones metódicos, el tiempo-espacio y la historia misma escrita por Occidente, como ejemplo de que son los vencedores (el lenguaje masculino no es ninguna casualidad en este caso) quienes cuentan la historia, deben ser replanteados para impulsar un orden social que posibilite la sobrevivencia de la especie y del planeta a futuro. 
categorialmente de lo que nos ocurre, y esto ya no es solo en la ciencia social latinoamericana, sino en la ciencia social occidental u occidentalizada, que se muestra incapaz de dar cuenta de lo que ocurre con el evidente estado de descomposición del orden estructurado por la modernidad. Para ello hemos intentado pensar en las formas diversas en las que opera la colonialidad para imposibilitarles a los pueblos colonizados nombrar su entorno, comprenderlo e interactuar con él desde lo que comenzamos a pensar y denominar en tanto colonialidad del color.

No pretendemos, de manera ingenua y arrogante, asignarle un nuevo nombre a cada verde con el que interactuamos. Nos parece, eso sí, oportuno pensar en un itinerario básico desde nuestra cotidianidad que apunte a ir más allá del horizonte de sentido contenido en la modernidad.

\section{La imposición de una realidad en verde monocromático}

Con el avistamiento de tierra «efectuado» el 12 de octubre de 1492 se dio inicio a un largo proceso de dominio colonial en el territorio conocido en la actualidad como América. A partir de este momento particular se dio inicio al sistema-mundo moderno que gira alrededor de la Europa latino-germánica por primera vez en la historia de la humanidad.

Con la llegada de los $d z u l e{ }^{15}$ las poblaciones autóctonas modificaron sus conceptos de memoria, identidad y tradición como resultado de la transformación de las relaciones comunitarias que estos habían desarrollado a través de una práctica milenaria.

Las relaciones comunitarias desarrolladas entre los distintos pueblos autóctonos del Nuevo Mundo se desarrollaban a través de luchas por la hegemonía política en la que el conocimiento y los saberes adquiridos les permitían comprender el mundo que habitaban y relacionarse con este, de manera que estos eran protegidos con recelo por parte de «los pipiltin -los nobles nahuas. Aquel saber señalaba modos de vida, costumbres por mantener, herencias por transmitir (...) aquello daba origen a un sistema educativo

15 Traducción del idioma maya al castellano realizada por Antonio Mediz Bolio en el año 1941, en este caso, en el libro de Chilam Balam de Chumayel. Con esta palabra los «indígenas» nombraban a los colonizadores. Este relato es testimonio de cómo se llevó a cabo la etapa de imposición del dogma religioso cristiano. 
con un desarrollo único. En los calmecac - Templo/escuela — habían sabios llamados poseedores de los libros de pinturas ${ }^{16}{ }_{»}$ (Gruzinski, 1991, p. 17).

Basado en fuentes exploradas mediante la antropología y la semiología, el historiador francés Serge Gruzinski trató de estudiar y crear un acercamiento a las transformaciones que se llevaron a cabo en los modos de expresión de lo que hoy llamamos México, del período prehispánico. El autor demostró cómo la educación, el saber y el manejo de la tradición eran protegidos por las comunidades autóctonas y cómo estas recurrían al uso del pigmento y del tlapalli — palabra náhuatl que se traduce a color - para reunir adecuadamente en sus representaciones visuales las relaciones, las prácticas y los simbolismos, que estaban ligados a la carga de color para asignarles un grado de significancia y connotación.

La pictografía era esencial para estas comunidades, que poseían una enumeración de los principales colores y cómo estos repercutían en la interpretación de su cosmovisión.

El origen del cromatismo náhuatl nos muestra cómo el color fue pieza clave en la definición de las deidades.

Xiuhtecuhtli —El Señor Azul—, es el nombre del dios más antiguo e importante, el dios del fuego (...) se representaba con este color. La cosmogonía náhuatl de los cuatro colores perduró por largo tiempo y aparece, también, en la leyenda de la creación del mundo que se desdoblan y confunden una con otra: El Tezcatlipoca negro - Norte- es el espejo que adivina a los hombres, el Tezcatlipoca azul — Sur- es siniestro. Asimismo aparece el Tezcatlipoca blanco - Oeste- y en el cuarto punto, entre el maíz verde, el Tezcatlipoca rojo —Este—, que es Xipe Totec (Ferrer, 1999, p. 217-218).17

Este fragmento de la producción de Eulalio Ferrer da cuenta de lo importante que era el color y que este mismo se personificaba; más que solo ser un recurso pictórico, trascendía y se convertía en una pieza clave de cómo giraba el mundo náhuatl. Percepciones y anotaciones que llegarían

16 El término pintura tal vez no sea el más adecuado para llamar a los pictogramas e ideogramas que mostraban los códices nahuas, ya que este concepto es meramente occidental y parece tratar de nombrar lo que parece por nuestra forma de pensar y el bagaje que se tenga, es también un tipo de apropiación del sujeto en cuanto a su entorno.

17 Ver anexo «Colores prehispánicos en la propia lengua náhuatl», de Eulalio Ferrer (1999). 
a ser destruidas por la desconfianza de parte de los padres franciscanos para contribuir a «evangelizar»; estos — los libros «pinturas»- representaban «el error y el engaño del demonio» (Gruzinski, 1991). La destrucción de los templos y los ídolos constituyó el objetivo primordial de los años 1520 a 1530, los franciscanos, seguidos por otras órdenes mendicantes, también confiscaron todas las pinturas que les parecían contrarias a la fe, «todo lo que es ceremoniático y sospechoso quemamos» (Gruzinski, 1991). Con frecuencia estas pinturas eran destruidas sin ninguna distinción, se dedicaron a la censura y la abolición de estas (Gruzinski, 1991). La religión católica se impuso y el dios del cristiano salió invicto. Sus creyentes y devotos se dedicaron a la destrucción de panteones y deidades sin importar ni pensar en el impacto existencial que eso tendría en los pueblos autóctonos.

Los códices muestran que existió, y sigue vigente, el conocimiento del pigmento, aspecto que no era ignorado y del cual solo algunos eran dignos, tanto de interpretar gráficamente como de conocer su cultura. Pero con la demolición de sus cosmogonías, la quema de su producción cultural y todo aquello que mostraba una forma del pensar comunitario se impuso una forma única de ver el mundo y entorno que habitamos. Las dinámicas de adaptación y resistencia con que se ha impuesto la modernidad son correspondientes con la aprehensión de una realidad y visión de mundo específica, pero que no ha significado el colapso de las relaciones comunitarias que han tenido que recurrir incluso a la clandestinidad, donde representaban gráficamente lo que recordaban gracias a la tradición oral.

En 1676, el físico Isaac Newton probó experimentalmente que la luz solar blanca se descompone, valiéndose de un prisma triangular, en los colores del espectro luminoso. ${ }^{18}$ Ya con anterioridad Aristóteles había trabajado este tema con la conformación de todos los colores con la mezcla de cuatro matices — tierra, fuego, agua y aire-, y, entre los siglos XVIII y XIX, Johann Wolfgang von Goethe propuso estrictamente la primera teoría del color y un interés en el impacto psicológico que estos tienen.

Para la producción del color se requiere luz y tinieblas, claro y oscuro (...) luz y no luz. Ante todo nos surge de la luz un color al que llamamos amarillo, y otro de las tinieblas al que designamos con la palabra azul. Cuando los dos

18 Según Itten (1960), este espectro contiene todos los colores principales, excepto el color púrpura. 
se mezclan, en su estado más puro, producen un tercero, al que nombramos verde, y en combinación con el rojo se crean los seis colores esenciales (Pawlik, 1996, p. 29).

Posterior a Goethe, múltiples autores han creado conclusiones y observaciones sobre esta teoría, entre ellos podemos mencionar a Philipp Otto Runge, Delacroix, Von Bezold, Johannes Itten y Paul Klee, docente de Bauhaus; todos occidentales. La caracterización del color se trata entonces como un aspecto filosófico-conceptual en Occidente, aun cuando los pueblos autóctonos ya dominaban el uso del pigmento, el cual no era arbitrario, y manejaban una especie de diagramación, elección e incluso grosor de línea que guardaba relación con el color.

Esta observación queda en evidencia si utilizamos la enumeración de colores y su respectivo nombre ante las distintas tonalidades que pueden resultar, ya sea por saturación, luminosidad o temperatura, este es un largo estudio de clasificación, y en muchos casos este tipo de jerga especializada es utilizada y enseñada en academias de arte occidentales y/u occidentalizadas, más específicamente por pintores. Un ejemplo de lo anterior es la imposición de la noción de un verde que poco o nada tiene de relación con las formas de concebir la realidad e interactuar con ella que tienen los pueblos autóctonos, caribeños, amerindios o mesoamericanos: «Verde cadmio (...) Verde bronce, Verde cobalto, Verde de Schweinfurt, Verde francés, Verde hooker, Verde militar, Verde París»(Heller, 2004, p. 104).

Lo anterior da cuenta, como señaló Wallerstein (2007), de la imposición de los «países centrales» en la actualidad. Por otra parte los sistemas operativos incorporados al uso del Pantone Matching System, que consiste en un sistema de identificación cromática, comparación y comunicación del color para las artes gráficas. Esta compañía definió una paleta de colores determinada que se concreta en las populares Guías Pantone, que lo que hacen es asignar a un color una numeración y unas siglas en función de la superficie o material en el que se vaya a aplicar. Claramente esto no solo aplica al campo del color, sino más bien a todo lo que nos rodea, nos dedicamos a la reproducción de este plan-mundo con ideas y planteamientos que en su gran mayoría provienen de lejanas fronteras y que no corresponden al espacio que habitamos. 
Los nombres de las ciudades que constituimos en la actualidad dejan en evidencia un legado colonial a lo largo de toda América Latina: San Rafael, San José, San Pedro, San Antonio, Santa Marta, etc., todo parte del mismo principio impositivo y es claro que nombrar es otra de las variadas formas de apropiación. Partiendo de lo anterior, otra arista de la imposición colonial que desplaza los templos y espacios de los pueblos autóctonos, en donde su organización arquitectural deja rastros de propósitos ajenos: la Iglesia cristiana al centro, al frente una plaza, que tal vez ahora será un parque, y a los alrededores centros policiacos; el papel de la Iglesia cristiana desde la llegada de Occidente a estas tierras ha tenido mucha importancia, por eso su posicionamiento en la población debía ser central, y resulta curiosa la función de las campanas, ya que estas en tiempos de «evangelización» se emplean para llamar al pueblo, el cual era un llamado casi obligatorio, ante el que la desobediencia era castigada incluso con la vida.

El pensamiento abstracto occidental como lo conocemos en la actualidad se fundó en Grecia.

Éste lleva a la autonomía de las formas y contenidos, es esencialmente promovida, a más de por las experiencias y aventuras de la colonización y sus circunstancias, por los medios y métodos de la economía monetaria. El carácter abstracto de los medios de cambio, la reducción de los diversos bienes a un común denominador, la división de bienes en los dos actos independientes de la compra y de la venta son factores que acostumbran a los hombres al pensamiento abstracto y los familiarizan con la idea de una misma forma con diversos contenidos y de un contenido igual con formas cambiantes (Hauser, 1994, p. 106-107).

Una vez que el hombre occidental distinguió entre contenido y forma - aseveración a la que los pueblos autóctonos ya se habían acercado-, no se alejó de la concepción de la autonomía de ambos, y de ahí el desarrollo de ideas y pensamientos en tanto balance; la creación del areté por parte de la aristocracia con pretensiones de mantener un límite clasista, la idea de perfección y la de belleza griegas son rasgos que se solidificaron posteriormente en Occidente.

A través de la imposición de la nomenclatura de los colores se logra mostrar cómo estos legados griegos se mantienen en nuestra cotidianidad 
como legado del orden greco-romano-latino-americano que integramos en la actualidad. Una moneda, un sistema laboral desigual y los procesos de producción, distribución y comercialización impulsados sobre la imposición de los pueblos autóctonos se constituyen como la base de la estructura económica que le permitió a Occidente consolidar un tipo de racionalidad, un tipo de economía sobre la base de la esclavitud.

\section{Rupturas urgentes más allá del color. Aperturas}

La necesidad de pensar en un tipo de racionalidad más allá que la contenida en el proyecto de la modernidad pasa por renombrar la belleza exuberante que rodea nuestro entorno tropical húmedo mesoamericano, el cual se manifiesta en una gama policromática de verdes que exigen ser nombrados.

La posibilidad de nombrar nuestro entorno sugiere una ruptura con las formas occidentalizadas de concebir el mundo, entendido este en tanto planeta Tierra dotado de recursos a disposición del «desarrollo», para trascender, metafísicamente si se quisiera, en una humanidad que es parte de la inmensidad del cosmos. Esto plantea el reto lingüístico de repensar la estructura del idioma, al tiempo que repensamos las relaciones sociales y comunitarias para diseñar horizontes de sentido donde la vida del planeta y de la humanidad tengan posibilidad de futuro.

La racionalidad de dominio incorporada en el proyecto de la modernidad le ha otorgado a la visión un lugar privilegiado sobre los otros sentidos, marcando una diferencia significativa respecto a la forma de interacción comunitaria de los pueblos autóctonos del Anáhuac, el Abya-Yala y el Tawantinsuyu con la naturaleza, al tiempo que ha impuesto hegemónicamente un tipo de relación social de la especie humana con el planeta. La teoría del color nació no solo para poder nombrar cada tonalidad que nos rodea, que percibimos visualmente y creamos a partir de la pigmentación, sino para controlar las formas de interacción comunitarias con la inmensidad del cosmos.

La imposición de una única razón, la que está contenida en la modernidad, le ha imposibilitado a la humanidad utilizar el espectro multi-sensorial del que la misma dispone, lo cual, según nuestro entender, atiende a una dimensión jerárquica de la colonialidad, de tipo sensorial, que ha impuesto 
una única manera de interacción de la humanidad con su entorno, implantando una única mirada acerca de él, una forma de nombrarlo, relacionarse y/o dominarlo. De esta forma la designación conceptual del color pasa a través de la mirada, mas no es solo con ella que se transforma la realidad, ya que para ello se requieren manos y pies, voz y olfato y mucha capacidad de escucha. Lo anterior deja claro que el reto prospectivo exige la dotación de nuevas formas que permitan al resto de los sentidos, además de la vista, jugar un papel en la designación del color, la concepción de la realidad y la posibilidad de transformarla, lo que implica pensar más allá de la modernidad. Esto implica el desarrollo de una agenda política cuyo sentido esté en la descolonización del color.

Nuestro interés por problematizar las formas de nombrar el verde es reflejo de nuestra incapacidad de pensar nuestro entorno en sentido amplio, en su dimensión viva, lo que sugeriría establecer un tipo de relacionamiento sujeto-sujeto con quien cotidianamente nos alimenta y nos ha permitido la vida. Lo anterior sugiere pensar, de manera problemática, los linderos y las posibilidades del tipo de racionalidad contenida en la modernidad, al tiempo que pensamos las fronteras del idioma colonial, en nuestro caso el español, para poder nombrar con propiedad el entorno que habitamos.

El proyecto sugiere identificar a la racionalidad moderna como una entre otros tipos de racionalidad, lo que sugiere identificar posibilidades de libertad, de creación, solo viables en la medida en que nuestro entorno continúe expresándose en una amplia gama de verdes, para lo cual el proceder de dominio con que actúa la lógica moderna debe permitirnos pensar más allá de esta forma de interacción de la especie con ella misma y el planeta. Para poder renombrar nuestro entorno lo primero que habrá que garantizar es la reproducción de la vida. Este último parece ser, en la actualidad, el principal reto de la especie. 


\section{ANEXO}

Colores Prehispánicos. El Color entre los Pueblos Nahuas

\begin{tabular}{|c|c|}
\hline Amarillo & Zacatazcalli \\
\hline Amarillo oscuro & Tecozahuitl \\
\hline Amarillo intenso & Coztic \\
\hline Azul & Xiuhuitl \\
\hline Azul agua & Toxpalatl \\
\hline Azul claro & Texotli \\
\hline Azul manchado & Cuitlatexotli \\
\hline Azul oscuro & Matlalli \\
\hline Azul turquesa & Tlaliac \\
\hline Blanco & Iztac \\
\hline Blanco grisáceo & Tizatl \\
\hline Blanco moteado & Chiotl \\
\hline Café claro & Quapachtli \\
\hline Morado & Cacamoliuhqui \\
\hline Morado oscuro & Yapalli \\
\hline Negro & Tliltic \\
\hline Negro intenso & Huizache \\
\hline Negruzco & Yayauhqui \\
\hline Rojo & Tlatlauhqui \\
\hline Rojo vivo & Cuezalli \\
\hline Rosa & Xochipalli \\
\hline Rosado morado & Xocoatole \\
\hline Verde & Xiuhuitl \\
\hline Verdeazul & Chalchíhuitl \\
\hline Verde claro & Quiltic \\
\hline Verde medio & Nochtli \\
\hline Verde intenso & Quilpalli \\
\hline Verde oscuro & Matlaltic \\
\hline Violeta & Matlaxóchitl \\
\hline
\end{tabular}

Fuente: Ferrer, E (1999). Los Lenguajes del Color. España: Fondo Cultura Económica de España. 


\section{Referencias}

Calvet, J. (2005). Lingüística y colonialismo. Breve tratado de glotofagia. Argentina: Fondo de Cultura Económica.

Dussel, E. (2011). El primer debate filosófico de la modernidad. En E., Dussel, E., Mendieta y C., Bohórquez (eds.), El pensamiento filosófico latinoamericano, del Caribe y "Latino" (1300-2000) [pp. 56-66]. México: Editorial Siglo XXI.

Ferrer, E. (1999). Los lenguajes del color. España: Fondo Cultura Económica de España.

Grosfoguel, R. (2011). La descolonización del conocimiento: diálogo crítico entre la visión descolonial de Franntz Fanon y la sociología descolonial de Boaventura de Sousa Santos. Recuperado de: http://www.cidob.org/en/publications/monographs/monographs/ formas_otras_saber_nombrar_narrar_hacer

Gruzinsky, S. (1991). La colonización de lo imaginario, sociedades indígenas y occidentalización en el México español. Siglos XVI-VIII. México: Fondo de Cultura Económica.

Hauser, A. (1994). Historia social de la literatura y del arte. Colombia: Grupo Editorial Quinto Centenario S.A.

Heller, E. (2004). Psicología del color. Cómo actúan los colores sobre los sentimientos y la razón. España: Editorial Gustavo Gili, S.L.

Marx, K. (1976). Cartas. Marx a Pavel Vasilievich Annenkov (Bruselas, 28 de diciembre de 1846). En K., Marx y F., Engels, Obras escogidas (pp. 531-542). Moscú: Editorial Progreso.

Pawlik, J. (1996). Teoría del color. España: Paidós Ibérica.

Rendón, J. (2012). Hegel, crítica del Estado moderno. México: Editorial Siglo XXI.

Wallerstein, I. (2007). Análisis de Sistema-Mundo. México. Editorial Siglo XXI. 